\title{
VERY STRONG MULTIPLICATION IDEALS AND THE IDEAL $\theta(I)$ OVER A COMMUTATIVE SEMIRING
}

\author{
Shahabaddin Ebrahimi Atani and Reza Ebrahimi Atani \\ University of Guilan, Iran
}

\begin{abstract}
Let $R$ a commutative semiring with identity. An ideal $I$ is called a multiplication ideal if every ideal contained in $I$ is a multiple of $I$. We consider the associated ideal $\theta(I)$. It is proved that the strong ideal $\theta(I)$ is important in the study of multiplication ideals. Among various applications given, the following results are proved: if $I$ is a faithful very strong multiplication ideal, then the strong ideal $\theta(I)$ is an idempotent ideal of $R$ such that $\theta(\theta(I))=\theta(I)$, and every secondary representable ideal of $R$ which is also a very strong multiplication ideal is finitely generated.
\end{abstract}

\section{INTRODUCTION}

For potential applications we note that ideals over semiring are important in studying the properties of the semiring, and the latter arise in diverse areas of applied mathematics, including optimization theory, automata theory, mathematical modeling and parallel computation systems. This paper is devoted to an exploration of how ideal-theoretic considerations in commutative semirings impact the multiplicative behavior of those elements of the semiring that have additive inverses in the semiring. The general question as to the algebraic nature of these so-called "zero-sums" of a semiring is one of the most central in the theory of semirings. The idea of investigating a mathematical structure via its representations in simpler structures is commonly used and often successful. The representation theory of semirings has developed greatly in the recent years. It is an area which is very firmly based on the detailed understanding of examples, and there are many powerful techniques for investigating the representations of particular semirings and for relating

2010 Mathematics Subject Classification. 16 Y60.

Key words and phrases. Very strong multiplication ideals, ideal $\theta(I)$, secondary ideals. 
the representations of one semiring to another. One of the aims of the modern representation theory of semirings is to solve "zero-sum" problems for subcategories of semirings. The reader is referred to [10] and [11] for a detailed discussion of "zero-sum" problems and useful computational reduction procedures.

Let $R$ be a commutative semiring with non-zero identity. An ideal $I$ of $R$ is called a multiplication ideal of $R$ whenever $J$ is an ideal of $R$ with $J \subseteq I$, then there exists an ideal $K$ of $R$ such that $J=K I([1,2,4])$. In this case we can take $K=(J: I)=\{r \in R: r I \subseteq J\}$. The main part of this paper is devoted to extending some basic results of multiplication ideals from the theory of rings to theory of semirings (see Sections 2, 3 and 4).

For the sake of completeness, we state some definitions and notations used throughout. A commutative semiring $R$ is defined as an algebraic system $(R,+,$.$) such that (R,+)$ and $(R,$.$) are commutative semigroups, connected$ by $a(b+c)=a b+a c$ for all $a, b, c \in R$, and there exists $0 \in R$ such that $r+0=r$ and $r 0=0 r=0$ for each $r \in R$. In this paper all semirings considered will be assumed to be commutative semirings. A subset $I$ of a semiring $R$ will be called an ideal if $a, b \in I$ and $r \in R$ implies $a+b \in I$ and $r a \in I$. A subtractive ideal (= $k$-ideal) $K$ is an ideal such that if $x, x+y \in K$ then $y \in K$ (so $\{0\}$ is a $k$-ideal of $R$ ). A prime ideal of $R$ is a proper ideal $P$ of $R$ in which $x \in P$ or $y \in P$ whenever $x y \in P$. If $I$ is an ideal of $R$, the radical $I$, denoted by $\operatorname{rad}(I)$, is the set of all $x \in R$ for which $x^{n} \in I$ for some positive integer $n$. This is an ideal of $R$, contains $I$ and is the intersection of all prime ideals of $R$ that contain $I$ ([3]). An ideal $I$ of a semiring $R$ is called a partitioning ideal (=Q-ideal) if there exists a subset $Q$ of $R$ such that $R=\cup\{q+I: q \in Q\}$ and if $q_{1}, q_{2} \in Q$ then $\left(q_{1}+I\right) \cap\left(q_{2}+I\right) \neq \emptyset$ if and only if $q_{1}=q_{2}$. Allen ([3]) has presented the notion of $Q$-ideal $I$ in the semiring $R$ and constructed the quotient semiring $R / I$ (also $[4,5,8-10]$ ). The following lemma is well known.

LEMMA 1.1. Let $R$ be a semiring $R$. If $\left\{I_{i}\right\}_{i \in \Lambda}$ is a collection of ideals of $R$, then $\sum_{i \in \Lambda} I_{i}$ and $\bigcap_{i \in \Lambda} I_{i}$ are ideals of $I$.

\section{VERY STRONG MULTIPLICATION IDEALS}

In this section we list some basic properties concerning very strong multiplication ideals. We begin the key definition of this paper.

Definition 2.1. An ideal $I$ of a semiring $R$ is said to be a strong ideal if for each $a \in I$ there exists $b \in I$ such that $a+b=0$.

ExAmple 2.2. Let $R=\{0,1,2, \ldots, 20\}$, and define $a+b=\max \{a, b\}$, $a . b=\min \{a, b\}$ for each $a, b \in R$. Then $(R,+,$.$) is easily checked to be a$ commutative semiring with 20 as identity. Let $J_{4}$ denote the ring of integers modulo 4. Let $J_{4} \oplus R=\left\{(a, b): a \in J_{4}, b \in R\right\}$ denote the direct sum of 
semirings $J_{4}$ and $R$. Then $J_{4} \oplus R$ is a commutative semiring. An inspection will show that $I_{0}=\left\{(a, 0): a \in J_{4}\right\}$ is a proper strong ideal in $J_{4} \oplus R$ and $I_{10}=\{(0, n): n \leq 10\}$ is a proper ideal of $J_{4} \oplus R$ which is not a strong ideal.

Let $R$ be a semiring. An ideal $P$ of $R$ is called a strong maximal $k$-ideal if it is maximal in the lattice of strong $k$-ideals of $R$.

Lemma 2.3. Let $R$ be a semiring with $1 \neq 0$. Then $R$ has at least one strong maximal $k$-ideal.

Proof. Since $\{0\}$ is a proper strong $k$-ideal of $R$, the set $\Delta$ of all proper strong $k$-ideals of $R$ is not empty. Of course, the relation of inclusion, $\subseteq$, is a partial order on $\Delta$. Now $\Delta$ is easily seen to be inductive under $\subseteq$, so by Zorn's Lemma $\Delta$ has a maximal element, i.e., $R$ has a proper strong maximal $k$-ideal.

THEOREM 2.4. Let $I$ be a proper strong $Q$-ideal of a semiring $R$. Then there exists a strong maximal $k$-ideal $M$ of $R$ with $I \subseteq M$.

Proof. Since $R / I$ is non-trivial, and so, by Lemma 2.3, has a strong maximal $k$-ideal $L$, which, by [5, Theorem 2.3], will have to have the form $M / I$ for some $k$-ideal $M$ of $R$ with $I \subseteq M$. It now follows from [5, Theorem $2.14]$ that $M$ is a $k$-maximal ideal of $R$. It remains to show that $M$ is a strong ideal of $R$. Let $a \in M$. Then $a=q+c \in q+I$ for some $c \in I$. Assume that $q_{0}$ is the unique element in $Q$ such that $q_{0}+I$ is the zero in $R / I$. Since $M$ is a $k$-ideal of $R$, we must have $q \in M \cap Q$, so $q+I \in M / I$. By assumption, $(q+I) \oplus\left(q^{\prime}+I\right)=q_{0}+I=I$ for some $q^{\prime}+I \in M / I$, where $q+q^{\prime}+I \subseteq I$; hence $q+q^{\prime}+e=f$ for some $e, f \in I$. As $f+c \in I$, there is an element $f^{\prime} \in I$ such that $f+c+f^{\prime}=0$, so $a+\left(q^{\prime}+e+f^{\prime}\right)=c+f+f^{\prime}=0$, as required.

LEMma 2.5. Let $I$ be a finitely generated ideal of a semiring $R$, and let $J$ be a strong ideal in $R$ such that $J I=I$. Then $(1+t) I=0$ for some $t \in J$.

Proof. Let $I=<x_{1}, x_{2}, \ldots, x_{n}>$. We use induction on $n$. Consider first the case in which $n=1$. Here we have $<x_{1}>=J<x_{1}>$. So $x_{1}=s x_{1}$ for some $s \in J$; hence there is an element $s^{\prime} \in J$ such that $s+s^{\prime}=0$ and hence $\left(1+s^{\prime}\right) x_{1}=s x_{1}+s^{\prime} x_{1}=0$. It follows that $\left(1+s^{\prime}\right) I=0$. We now turn to the inductive step. Assume, inductively, that $n=k+1$, where $k \geq 1$, and that the result has been proved in the case where $n=k$. Then we must have $\left.\left.(1+a)(1+b) I=(1+a+b+a b)\left(<x_{1}, \ldots, x_{k}\right\rangle+<x_{k+1}\right\rangle\right)=0$ for some $a, b \in J$, so $(1+t) I=0$, where $a+b+a b=t \in J$, as needed.

Proposition 2.6. Let $I$ be a strong finitely generated ideal of a semiring $R$, and let $J$ be a strong ideal in $R$ such that $J I=I$. Then $R=J+\operatorname{ann}(I)$.

Proof. By Lemma 2.5, There exists $t \in J$ and $c \in \operatorname{ann}(I)$ such that $1+t=c$, so $r+r t=r c$ for all $r \in R$. By assumption, there is an element $d \in J$ such that $r t+d=0$; hence $r=r c+d \in J+\operatorname{ann}(I)$, as required. 
Let $R$ be a semiring. An ideal $I$ of $R$ is said to be Hopfian if each $R$ epimorphism $f: I \rightarrow I$ is isomorphism.

Proposition 2.7. Let $R$ be a semiring. If $I$ is a multiplication ideal of $R$, then $I$ is Hopfian.

Proof. Let $f: I \rightarrow I$ be a epimorphism. By assumption, there exists an ideal $J$ of $R$ such that $K=\operatorname{Ker}(f)=J I$. Hence $0=f(K)=J f(I)=I J=$ $K$, as needed.

Proposition 2.8. Let I be an ideal of a semiring $R$. Then I is multiplication if and only if $I \cap J=I(J: I)$ for every ideal $J$ of $R$.

Proof. Suppose that $J \subseteq I$ is an ideal of $R$. Then $J=I \cap J=I(J$ : $I)=J I$. Conversely, assume that $I$ is a multiplication ideal of $R$. Let $K$ be any ideal of $R$. Then $I \cap K \subseteq I$ and so there exists an ideal $A$ of $R$ such that $I \cap K=A I$. Therefore, $A \subseteq(I \cap K: I) \subseteq(K: I)$ and then $I \cap K=A I \subseteq I(K: I)$. On the other hand, clearly, $I(K: I) \subseteq I \cap K$, and so we have equality.

Proposition 2.9. An ideal $I$ of a semiring $R$ is multiplication ideal if and only if for each a in $I$ there exists an ideal $J$ of $R$ such that $R a=J I$.

Proof. The necessity is clear. For the sufficiency, suppose that for each $a \in I$ there exists an ideal $J$ of $R$ such that $R a=J I$. Let $K$ be an ideal of $R$ with $K \subseteq I$. For each $x \in K$ there exists an ideal $J_{x}$ such that $R x=J_{x} I$. Let $J=\sum_{x \in K} J_{x}$. Then $K=\sum_{x \in K} R x=\sum_{x \in K} J_{x} I=J I$. It follows that $I$ is a multiplication ideal.

Definition 2.10. Assume that $P$ is a strong maximal $k$-ideal of a semiring $R$ and let $I$ be an ideal of $R$.

(i) We say that $I$ is $P$-cyclic provided there exists $p \in P$ and $a \in I$ such that $(1+p) I \subseteq R a$.

(ii) We say that $\bar{I}$ is $P$-torsion if $I=T_{P}(I)=\{a \in I:(1+p) a=$ 0 for some $p \in P\}$.

The definition 2.10 is the same as that introduced by Z. El-Bast and P. F. Smith in [4].

Lemma 2.11. Assume that $P$ is a strong maximal $k$-ideal of a semiring $R$ and let $I$ be an ideal of $R$. Then $T_{P}(I)$ is a subideal of $I$.

Proof. Let $a, b \in T_{P}(I)$ and $r \in R$. Then there exist $p_{1}, p_{2} \in P$ such that $\left(1+p_{1}\right) a=0$ and $\left(1+p_{2}\right) b=0$, so $\left(1+p_{1}\right)\left(1+P_{2}\right)(a+b)=\left(1+p_{1}\right)(r a)=0$, and the proof is complete.

Definition 2.12. Let $R$ be a semiring. A strong ideal $I$ of $R$ is called a strong multiplication ideal of $R$ whenever $J$ is an ideal of $R$ with $J \subseteq I$, then there exists a strong ideal $K$ of $R$ such that $J=K I$. 
Lemma 2.13. Let $R$ be a semiring. Then the following hold:

(i) If $I$ is an strong ideal of $R$, then $R x$ is a strong ideal of $R$ for every $x \in I$.

(ii) A finite sum of strong ideals of $R$ is a strong ideal of $R$.

(iii) If $I$ and $J$ are strong ideals of $R$, then $I J$ is a strong ideal of $R$.

Proof. The proof is straightforward.

THEOREM 2.14. Let $I$ be a strong multiplication ideal of a semiring $R$. Then for every strong maximal $k$-ideal $P$ of $R$ either $I=T_{P}(I)$ or $I$ is $P$ cyclic.

Proof. Let $P$ be a strong maximal $k$-ideal of $R$. Suppose $I=P I$. Let $a \in I$. Then $R a=J I$ for some strong ideal $J$ of $R$. Hence $R a=J I=J P I=$ $P a$ and $a=p a$ for some $p \in P$. By assumption, there exists $p^{\prime} \in P$ such that $p a+p^{\prime} a=\left(1+p^{\prime}\right) a=0$ and hence $a \in T_{P}(I)$. It follows that $T_{P}(I)=I$. Now suppose that $P I \neq I$. There exists $b \in I$ and $b \notin P I$. There is a strong ideal $K$ of $R$ such that $R b=K I$. Clearly, $K \not \subset P$. Since by Lemma $2.13, K+P$ is a strong ideal of $R$, we must have $K+P=R$, so $1=e+q$ for some $e \in K$ and $q \in P$. There exists $q^{\prime} \in P$ such that $q+q^{\prime}=0$; hence $1+q^{\prime} \in K$. It follows that $\left(1+q^{\prime}\right) I \subseteq R b$ and $I$ is $P$-cyclic.

Definition 2.15. Let $R$ be a semiring. A strong ideal $I$ of $R$ is called a very strong ideal of $R$ if $J$ is an ideal of $R$ with $J \subseteq I$ and $a \in I$, then the ideal $\{r \in R: r a \in J I\}$ is a strong $Q$-ideal.

Definition 2.16. Let $R$ be a semiring. A very strong ideal $I$ of $R$ is called a very strong multiplication ideal of $R$ whenever $J$ is an ideal of $R$ with $J \subseteq I$, then there exists a strong ideal $K$ of $R$ such that $J=K I$.

Compare the next theorem with [4, Theorem 1.2].

TheOrem 2.17. Let $I$ be an ideal of a semiring $R$. Then $I$ is a very strong multiplication ideal if and only if for every strong maximal $k$-ideal $P$ of $R$ either $I=T_{P}(I)$ or $I$ is $P$-cyclic.

Proof. If $I$ is a very strong multiplication ideal, then the result follows from Theorem 2.14. Conversely, suppose that for each strong maximal $k$ ideal $P$ of $R$ either $I=T_{P}(I)$ or $I$ is $P$-cyclic. Let $J$ be a subideal of $I$ and $K=(J: I)$. Clearly, $K I \subseteq J$. Let $y \in J$. Then by assumption, $L=\{r \in R: r y \in K I\}$ is a strong $Q$-ideal of $R$. Suppose $L \neq R$. Then by Theorem 2.4, there exists a strong maximal $k$-ideal $P^{\prime}$ of $R$ such that $L \subseteq P^{\prime}$. If $I=T_{P^{\prime}}(I)$, then there is an element $p^{\prime} \in P^{\prime}$ such that $\left(1+p^{\prime}\right) y=0 \in K I$; hence $\left(1+p^{\prime}\right) \in L \subseteq P^{\prime}$, which is a contradiction since $P^{\prime}$ is a strong maximal $k$-ideal. Thus by hypothesis, there exist $t \in P^{\prime}$ and $z \in I$ such that $(1+t) I \subseteq$ $R z$. It follows that $(1+t) J$ is a subideal of $R z$. Set $T=\{r \in: r z \in(1+t) J\}$. We show that $T z=(1+t) J$. Since the inclusion $T z \subseteq(1+t) J)$ is clear, we 
will prive the reverse inclusion. Let $x \in(1+t) J$. Then $x=(1+t) b$ for some $b \in J$. As $(1+t) J \subseteq R z$, we must have $(1+t) b=r z \in(1+t) J$, so $x=r z \in T z$, so we have equiality. Note that $(1+t) T I=T(1+t) I \subseteq T z \subseteq J$ and hence $(1+t) T \subseteq(J: I)=K$. Therefore, $(1+t)^{2} y \in(1+t)^{2} \bar{J}=(1+t) T z \subseteq K I$, so $(1+t)^{2} \in L \subseteq P^{\prime}$, a contradiction. Thus $L=R$ and $y \in K I$. It follows that $J=K I$ and $I$ is a very strong multiplication ideal.

Compare the next theorem with [4, Theorem 1.6].

THEOREM 2.18. Let I be a faithful ideal of a semiring $R$, and let $I$ be a very strong multiplication ideal. Then the following hold:

(i) $\bigcap_{i \in \Lambda}\left(I_{i} I\right)=\left(\bigcap_{i \in \Lambda} I_{i}\right) I$ for any non-empty collection of strong ideals $I_{i}(i \in \Lambda)$ of $R$.

(ii) For any subideal $J$ of $I$ and strong ideal $K$ of $R$ such that $J \subseteq K I$ there exists an ideal $L$ of $R$ with $L \subseteq K$ and $J \subseteq L I$.

Proof. Let $I_{i}(i \in \Lambda)$ be any non-empty collection of strong ideals of $R$. Set $J=\bigcap_{i \in \Lambda} I_{i}$. Clearly, $I J \subseteq \bigcap_{i \in \Lambda}\left(I_{i} I\right)$. For the reverse inclusion, assume that $x \in \bigcap_{i \in \Lambda}\left(I_{i} I\right)$. Then $K=\{r \in R: r x \in J I\}$ is a strong $Q$-ideal of $R$. Suppose $K \neq R$. Then by Theorem 2.4, there exists a strong maximal $Q$-ideal $P$ of $R$ such that $K \subseteq P$. Clearly, $x \notin T_{P}(I)$. For if $x \in T_{P}(I)$, then $(1+p) x=0 \in I J$ for some $p \in P$; hence $(1+p) \in K \subseteq P$, a contradiction. Therefore, $I$ is $P$-cyclic by Theorem 2.17. There exist $p \in P$ and $a \in I$ such that $(1+p) I \subseteq R a$. Then $(1+p) x \in \bigcap_{i \in \Lambda}\left(I_{i} a\right)$. For each $i \in \Lambda$, there is an element $a_{i} \in I_{i}$ such that $(1+p) x=a_{i} a$. Choose $j \in \Lambda$. Then for each $i \in \Lambda$, $a_{j} a=a_{i} a$. By assumption, $a_{i}+a_{i}^{\prime}=0$ for some $a_{i}^{\prime} \in I_{i}$; hence $a_{j} a+a_{i}^{\prime} a=0$. Now $(1+p)\left(a_{j}+a_{i}^{\prime}\right) I \subseteq\left(a_{j}+a_{i}^{\prime}\right) R a=0$ implies $(1+p)\left(a_{j}+a_{i}^{\prime}\right)=0$ since $I$ is faithful. Therefore, $a_{j}+p a_{j}+a_{i}^{\prime}+p a_{i}^{\prime}=0$, so $(1+p) a_{j}=(1+p) a_{i} \in I_{i}$; hence $(1+p) a_{j} \in J$. Thus $(1+p)^{2} x=(1+p)\left(a_{i} a\right) \in J I$. It follows that $(1+p)^{2} \in K \subseteq P$, a contradiction. So $K=R$; hence $x \in J I$, and (i) is proved.

(ii) Let $J$ be a subideal of $I$ and $K$ a ideal of $R$ such that $J \subseteq K I$. There exists a strong ideal $C$ of $R$ such that $J=C I$. Let $B=K \cap C$. Then $J=K I \cap C I=(K \cap C) I=B I$ by (i), as needed.

Compare the next result with [13, p. 231 Corollary].

Proposition 2.19. Let I be a faithful finitely generated ideal of a semiring $R$, and let $I$ be a very strong multiplication ideal. Then If $J$ and $K$ are very strong $k$-ideals of $R$ such that $J I \subseteq K I$, then $J \subseteq K$.

Proof. Let $a \in J$. Then by assumption, $T=\{r \in R: r a \in K\}$ is a strong $Q$-ideal of $R$. Suppose $T \neq R$. Then there exists a strong maximal $k$-ideal $P$ of $R$ such that $T \subseteq P$. If $P I=I$, then $(1+p) I=0$ for some $p \in P$ by Lemma 2.5, which is a contradiction since $I$ is faithful. Thus $I \neq P I$ and by Theorem 2.17, there exist $b \in I$ and $q \in P$ such that $(1+q) I \subseteq R b$. In 
particular, $(1+q) a b \in J I \subseteq J b$, so that there is an element $c \in J$ such that $(1+q) a b=c b$. There exists $a^{\prime} \in J$ such that $a+a^{\prime}=0$, so $\left(c+(1+q) a^{\prime}\right) b=0$. But $(1+q) \operatorname{ann}(b) \subseteq \operatorname{ann}(I)=0$; hence $(1+q)\left((1+q) a^{\prime}+c\right)=0$ and this implies that $(1+q)^{2} a \in J$ (since $(1+q)^{2} a+(1+q)^{2} a^{\prime}=0$ and $J$ is a $k$-ideal) so that $(1+q)^{2} \in T \subseteq P$, a contradiction. So $T=R$ and hence $a \in J$, as required.

Proposition 2.20. Let $P$ be a strong prime $k$-ideal of a semiring $R$ and $I$ a faithful very strong multiplication ideal of $R$. Let $a \in R, x \in I$ satisfy ax $\in P I$. Then $a \in P$ or $x \in P I$.

Proof. Let $a \notin P$. Then the ideal $K=\{r \in R: r x \in P I\}$ is a strong $Q$-ideal of $R$. Suppose $K \neq R$. Then there exists a strong maximal $k$-ideal $P^{\prime}$ of $R$ such that $K \subseteq Q$. Clearly, $x \notin T_{P^{\prime}}(I)$ (see the proof of Theorem 2.18). By Theorem 2.4, $I$ is $Q$-cyclic, that is, there exist $b \in I$ and $q \in P^{\prime}$ such that $(1+q) I \subseteq R b$. In particular, $(1+q) x=s b$ for some $s \in R$. Therefore we have $(1+q) a x \in(1+q) P I \subseteq P R b=P b$; hence $a s b=p b$ for some $p \in P$. By assumption, $p+p^{\prime}=0$ for some $p \in P^{\prime}$; hence $\left(a s+p^{\prime}\right) b=0$. Since $(1+q) \operatorname{ann}(b) I \subseteq R \operatorname{ann}(b) b=0$, we must have $(1+q)\left(a s+p^{\prime}\right)=0,(1+q) a s=(1+q) p \in P$. But $P \subseteq K \subseteq P^{\prime}$ so that $s \in P$ and $(1+q) x=s b \in P I$. Thus $(1+q) \in K \subseteq P^{\prime}$, which is a contradiction. It follows that $K=R$ and $x \in P M$, as required.

Theorem 2.21. Let $P$ be a strong prime $k$-ideal of a semiring $R$ and $I$ a faithful very strong multiplication ideal of $R$. If $P I \neq I$, then $P I$ is a strong prime subideal of $I$.

Proof. Apply Proposition 2.20.

\section{THE IDEAL $\theta(\mathrm{I})$}

In this section we study the ideal $\theta(\mathrm{I})$, where $R$ is a commutative semiring with identity and $I$ is a multiplication ideal of $R$.

REMARK 3.1. Let $I$ be an ideal of a semiring $R$.

(i) If $I$ and $J$ are finitely generated ideals of $R$, then $I J$ is a finitely generated ideal of $R$.

(ii) If $I$ is an ideal of $R$, then we define the subset $\theta(I)$ of $R$ as $\theta(I)=$ $\sum_{x \in I}(R x: I)$. Therefore, by Lemma 1.1, $\theta(I)$ is an ideal of $R$ (this definition is the same as that introduced by D. D. Anderson in [1]).

LemMA 3.2. Let $I$ be a multiplication ideal of a semiring $R$. Then $I=$ $I \theta(I)$. More generally, for any ideal $J \subseteq I, J=J \theta(I)$.

Proof. For $x \in I, R x=I(R x: I)$. Hence $I=\sum_{x \in I} R x=\sum_{x \in I} I(R x$ : $I)=I\left(\sum_{x \in I}(R x: I)\right)=I \theta(I)$. Moreover, since $J \subseteq I$, there exists an ideal $K$ of $R$ such that $J=K I$. Thus $J=K I=K(I \theta(I))=(K I) \theta(I)=J \theta(I)$. 
Compare the next result with [2, Lemma 2.1].

Proposition 3.3. Suppose that I is a strong multiplication ideal over a semiring $R$ such that $\theta(I)$ is a strong ideal. If $J$ is a finitely generated ideal of $R$ with $I \subseteq \theta(I)$, then $J I$ is finitely generated. Conversely, if $J$ is a strong ideal of $R$ with $J$ I finitely generated, then $J \subseteq \theta(I)$. In particular, if $r \in \theta(I)$, then $r I$ is finitely generated.

Proof. Let $J=<a_{1}, \ldots, a_{n}>$. Then there exist $x_{i} \in I(1 \leq i \leq n)$ such that $a_{i} \in\left(R x_{i}: I\right)$; hence $J \subseteq \sum_{i=1}^{n}\left(R x_{i}: I\right)$. Therefore, $I J \subseteq$ $\sum_{i=1}^{n} R x_{i}=K$. It follows from Lemma 3.2, Proposition 2.6 and Lemma 2.13 that $\theta(I) K=K$ and $R=\theta(I)+(0: K)$. There are elements $a \in \theta(I)$ and $b \in(0: K)$ such that $1=a+b$. Hence there exist $y_{1}, \ldots, y_{s} \in I$ such that $a \in \sum_{j=1}^{s}\left(R y_{j}: I\right)$; thus $R=(0: K)+\sum_{j=1}^{s}\left(R y_{i}: I\right)$. It follows that $I J=I R y_{1}+\ldots+I R y_{s}$ (since $\left.I J(0: K)=0\right)$; hence $I J$ is finitely generated by Remark 3.1. Conversely, Let $J$ be an ideal of $R$ and suppose that $I J$ is finitely generated. First we show that $I(0: I J) \subseteq(0: I)$. It suffices to show that for each $a \in I, b \in(0: I J), a b I=0$. As $b I J=0$, we must have $a b I=0$. Since $I J$ is finitely generated and $I J=\theta(I) I J$, so $R=\theta(I)+(0: I M)$ by Lemma 2.13 and Proposition 2.6. Hence $I=I \theta(I)+I(0: I J) \subseteq \theta(I)+(0: I) \subseteq \theta(I)$ because $(0: I) \subseteq \theta(I)$.

Compare the next theorem with [2, Theorem Corollary 2.2].

THEOREM 3.4. Suppose that $I$ is a strong multiplication ideal over a semiring $R$ such that $\theta(I)$ is a strong ideal. Then the following conditions are equivalent:

(i) I is finitely generated.

(ii) $\theta(I)=R$.

(iii) $\theta(I)$ is finitely generated.

Proof. (i) $\Longrightarrow$ (ii). Apply the second part of Proposition 3.3. (ii) $\Longrightarrow$ (iii). Clear. (iii) $\Longrightarrow$ (i). Set $J=\theta(I)$. Then by Proposition 3.3, $I=\theta(I) I$ is finitely generated.

THEOREM 3.5. Suppose that $I$ is a very strong multiplication ideal over a semiring $R$ such that $\theta(I)$ is a strong ideal. If $J$ is a strong ideal of $R$ with $J \subseteq \theta(I)$, then the following hold:

(i) $J+(0: I)=J \theta(I)+(0: I)$.

(ii) $\theta(I)=(\theta(I))^{2}+(0: I)$. In particular, If $I$ is faithful, then $(\theta(I))^{2}=$ $\theta(I)$.

Proof. (i) Since the inclusion $J \theta(I)+(0: I) \subseteq J+(0: I)$ is clear, we will prove the reverse inclusion. Let $c+a \in J+(0: I)$ for some $c \in J \subseteq \theta(I)$ and $a \in(0: I)$. Then $R c$ is a cyclic ideal of $R$ and $(R c) I=c I$ is finitely generated by Proposition 3.3. Hence $\theta(I) c I=c I$ gives $\theta(I)+(0: c I)=R$. 
Thus $c \theta(I)+c(0: I)=R c$. It follows from $c(0: c I) \subseteq(0: I)$ that $c \theta(I)+(0$ : $I)=(0: I)+R c$. Therefore, we have $R c+(0: I) \subseteq c \theta(I)+(0: I)$, so $c+a \in c \theta(I)+(0: I)$, and we have equality.

(ii) By (i), setting $I=\theta(I)$ gives $\theta(I)=\theta(I)+(0: I)=(\theta(I))^{2}+(0: I)$, as required

Given a very strong ideal $I$ of a semiring $R$, there are a number of very strong ideals associated with $I$ besides $\theta(I)$. By Lemma 1.1, $T(I)=\bigcap\{J+(0$ : $I): J$ is a very strong k-ideal of $R$ with $J I=I\}$ is an ideal of $R$ (see [2]). We next show that for $I$ a faithful very strong multiplication ideal of $R$, these two associated ideals coincide: $T(I)=\theta(I)$.

LEMMA 3.6. Let I be a faithful very strong multiplication $k$-ideal over a semiring $R$. Then the following hold:

(i) $a \in T(I) a$ for each $a \in I$.

(ii) $T(I)=(T(I))^{2}$.

Proof. (i) Let $T=T(I)$. By Theorem 2.18, $T I=(\bigcap J) I=\bigcap(J I)=T$. Then $R a=K I$ for some ideal $K$ of $R$. Thus $R a=K T I=T a$ and hence $a \in T a$.

(ii) $I=T I=T(T I)=T^{2} I$ implies $T=T^{2}$ by the definition of $T$.

Theorem 3.7. Suppose that $I$ is a faithful very strong multiplication $k$ ideal over a semiring $R$ such that $\theta(I)$ is a strong ideal. Then the following hold:

(i) $\theta(I)=T(I)$.

(ii) $\theta(I)=\theta(\theta(I))$.

Proof. (i) Let $I$ be a faithful very strong multiplication $k$-ideal of $R$. Now $\theta(I) I=I$, so $T(I) \subseteq \theta(I)$. By Theorem 3.5, $T(I)=T(I) \theta(I)$. For each $a \in I, T(I) R a=R a$ by Lemma 3.6. Hence $T(I)+(0: a)=R$. Now $(R a: I)(0: a) \subseteq(0: I)=0$, so $T(I)(R a: I)=(R a: I)$. Thus $T(I) \theta(I)=$ $T(I)\left(\sum_{a \in I}(R a: I)\right)=\sum_{a \in I} T(I)(R a: I)=\sum_{a \in I}(R a: M)=\theta(I)$. Hence $T(I)=\theta(I)$.

(ii) Since $I$ is faithful and $\theta(I) I=I$, we must have $\theta(I)$ is faithful. Hence by Theorem 3.5, $\theta(I)$ is a faithful idempotent multiplication $k$-ideal of $R$. Now $(\theta(I))^{2}=\theta(I)$ gives $T(\theta(I)) \subseteq \theta(I)$. So $\theta(\theta(I))=T(\theta(I)) \subseteq \theta(I) \subseteq \theta(\theta(I))$ and hence $\theta(\theta(I))=\theta(I)$.

\section{REPRESENTABLE IDEALS}

Let $R$ be a semiring. A non-zero ideal $I$ is secondary if for each $a \in R$, the endomorphism $\varphi_{a, I}$ (i.e., multiplication by $a$ in $I$ ) is either surjective or nilpotent. It is immediate that $\operatorname{rad}(\operatorname{ann}(I))=P$ is a prime ideal of $R$ (since if $a b \in \operatorname{rad}(\operatorname{ann}(I))=A$ with $a \notin A$, then $a^{n} b^{n} I=0$ for some $n$ 
and $a^{n} I=I$; hence $b^{n} I=0$. Therefore, $b \in A$ ), and $I$ is said to be $P$ secondary. An ideal $I$ is said to be secondary representable if it can be written as a sum $I=I_{1}+\ldots+I_{k}$ with each $I_{i}$ secondary, and if such a representation exists (and is irredundant) then the attached primes of $I$ are $\operatorname{Att}(I)=\left\{\operatorname{rad}\left(\operatorname{ann} I_{1}\right), \ldots, \operatorname{rad}\left(\operatorname{ann} I_{k}\right)\right\}($ see $[12])$.

Let $R$ be a semiring. An ideal $I$ is sum-irreducible if $I \neq 0$ and the sum of any two proper subideals of $I$ is always a proper subideal. We say that $R$ is Artinian if any non-empty set of $k$-ideals of $R$ has a minimal member with respect to set inclusion. This definition is equivalent to descending chain condition on $k$-ideals of $R$.

LEMma 4.1. Let $R$ be a semiring. Then a finite sum of P-secondary ideals is $P$-secondary.

Proof. Let $I=I_{1}+\ldots+I_{k}$, where for each $i(1 \leq i \leq k), I_{i}$ is $P$ secondary. Let $a \in R$. If $a \in P$, then there is a positive integer $n$ such that $a^{n} I_{i}=0$ for every $i$; hence $a^{n} I=0$. Similarly, if $a \notin P$, then $a I=I$. Thus $I$ is $P$-secondary.

THEOREM 4.2. Let $R$ be a semiring and let $I$ be a secondary representable multiplication ideal of $R$. Then every subideal $J$ of $I$ is secondary representable.

Proof. Let $I=\sum_{i=1}^{n} I_{i}$ be a minimal secondary representation of $I$ with $\operatorname{Att}(I)=\left\{P_{1}, P_{2}, \ldots, P_{n}\right\}$. Then $J=I K$ for some ideal $K$ of $R$ and $J=\sum_{i=1}^{n} K I_{i}$. It suffices to show that for each $i(1 \leq i \leq n), K I_{i}$ is $P_{i^{-}}$ secondary. Let $a \in R$. If $a \in P_{i}$, then $a^{m}\left(K I_{i}\right)=K\left(a^{m} I_{i}\right)=0$ for some $m$. If $a \notin P_{i}$, then $a\left(K I_{i}\right)=K I_{i}$, as required.

Compare the next theorem with [11, p. $36(5.2)]$.

TheOREM 4.3. Let $R$ be a semiring. Then every strong Artinian $k$-ideal $I$ of $R$ has a secondary representation.

Proof. First, we show that if $I$ is sum-irreducible, then $I$ is secondary. Suppose $I$ is not secondary. Then there is an element $r \in R$ such that $r I \neq I$ and $r^{n} I \neq 0$ for all positive integer $n$. By assumption, there exists a positive integer $k$ such that $r^{k} I=r^{k+1} I=\ldots$. Set $I_{1}=\operatorname{Ker} \varphi_{r^{k}, I}$ and $M_{2}=r^{k} I$. Then $I_{1}$ and $I_{2}$ are proper ideals of $I$. Let $x \in M$. Then $r^{k} x=r^{2 k} y$ for some $y \in I$. By assumption, $y+y^{\prime}=0$ for some $y^{\prime} \in I$; hence $r^{k} y+r^{k} y^{\prime}=0$, $r^{2 k} y+r^{2 k} y^{\prime}=0$ and $x=\left(x+r^{k} y^{\prime}\right)+r^{k} y$, where $x+r^{k} y^{\prime} \in I_{1}$ and $r^{k} y \in I_{2}$. Hence $I=I_{1}+I_{2}$, and therefore $I$ is not sum-irreducible. Next, suppose that $I$ is not secondary representable. Then the set of non-zero $k$-subideals of $I$ which are not secondary representable has a minimal element $J$. Certainly $J$ is not secondary and $J \neq 0$; hence $J$ is the sum of two strictly smaller subideals $J_{1}$ and $J_{2}$. By the minimality of $J$, each $J_{1}, J_{2}$ is secondary representable, and therefore so also is $J$, which is a contradiction. 
Proposition 4.4. Suppose that I is a strong multiplication ideal over a semiring $R$ such that $\theta(I)$ is a strong ideal. If $J$ is a P-secondary subideal of $I$, then there exists $a \in R$ such that $a \in \theta(I)$ and $a \notin P$. In particular, aI is finitely generated.

Proof. Suppose the opposite. Then $\theta(I) \subseteq P$. Let $x \in J$. Then by Lemma $3.2, R x=\theta(I) R x \subseteq P x \subseteq R x$, so $x=p x$ for some $p \in P$. There is a positive integer $m$ such that $p^{m} x=x=0$, which is a contradiction. Finally, $a I$ is finitely generated by Proposition 3.5.

THEOREM 4.5. Suppose that $I$ is a strong multiplication secondary representable ideal over a semiring $R$ such that $\theta(I)$ is a strong ideal. Then $I$ is finitely generated.

Proof. Let $I=\sum_{i=1}^{n} I_{i}$ be a minimal secondary representation of $I$ with $\operatorname{Att}(I)=\left\{P_{1}, P_{2}, \ldots, P_{n}\right\}$. By Proposition 4.4, for each $i(1 \leq i \leq n)$, there exists $a_{i} \in R$ such that $a_{i} \in \theta(I)$ and $a_{i} \notin P_{i}$. Then for each $i(1 \leq i \leq n)$, $a_{i} I=a_{i} I_{1}+\ldots+a_{i} I_{i-1}+I_{i}+a_{i} I_{i+1}+\ldots+a_{i} I_{n}$. Setting $a=\sum_{i=1}^{n} a_{i}$ gives $I=a I=a_{1} I+\ldots+a_{n} I$ is finitely generated by Proposition 3.5.

ThEOREM 4.6. Suppose that I is a strong multiplication Artinian k-ideal over a semiring $R$ such that $\theta(I)$ is a strong ideal. Then $I$ is finitely generated.

Proof. Apply Theorem 4.3 and Theorem 4.5.

ACKNOWLEDGEMENTS.

We would like to thank the referees for a number of useful comments and suggestions which have improved the paper.

\section{REFERENCES}

[1] D. D. Anderson, Some remarks on multiplication ideals, Math. Japon. 25 (1980), 463-469.

[2] D. D. Anderson and Y. Al-Shaniafi, Multiplication modules and the ideal $\theta(M)$, Commm. Algebra 30 (2002), 3383-3390.

[3] P. J. Allen, A fundamental theorem of homomorphisms for semirings, Proc. Amer. Math. Soc. 21 (1969), 412-416.

[4] Z. A. El-Bast and P. F. Smith, Multiplication modules, Commm. Algebra 16 (1988), $755-779$.

[5] S. Ebrahimi Atani, The ideal theory in quotients of commutative semirings, Glas. Mat. Ser. III 42(62) (2007), 301-308.

[6] S. Ebrahimi Atani, On primal and weakly primal ideals over commutative semirings, Glas. Mat. Ser. III 43(63) (2008), 13-23.

[7] S. Ebrahimi Atani, The zero-divisor graph with respect to ideals of a commutative semiring, Glas. Mat. Ser. III 43(63) (2008), 309-320.

[8] S. Ebrahimi Atani, An ideal-based zero-divisor graph of a commutative semiring, Glas. Mat. Ser. III 44(64) (2009), 141-153.

[9] R. Ebrahimi Atani and S. Ebrahimi Atani, Ideal theory in commutative semirings, Bul. Acad. Ştiinţe Repub. Mold. Mat. 2 (2008), 14-23. 
[10] J. S. Golan, The theory of semirings with applications in mathematics and theoretical computer science, Pitman Monographs and Surveys in Pure and Applied Mathematics 54, Longman Scientific and Technical, Harlow, 1992.

[11] U. Hebisch and U. J. Weinert Halbringe. Algebraische Theorie und Anwendungen in der Informatik, Teubner, Stuttgart, 1993.

[12] I. G. Macdonald, Secondary representation of modules over commutative ring, Symposia Mathematica XI, Academic Press, London, 1973, 23-43.

[13] P. F. Smith, Some remarks on multiplication modules, Arch. Math. (Basel) 50 (1988), 223-235.

S. Ebrahimi Atani

Department of Mathematics

University of Guilan

P.O. Box 1914, Rasht

Iran

E-mail: ebrahimi@guilan.ac.ir

R. Ebrahimi Atani

Department of Electrical Engineering

University of Guilan

P.O.Box 3756, Rasht

Iran

E-mail: rebrahimi@guilan.ac.ir

Received: 2.6.2009.

Revised: 24.1 .2010 . \& 2.2.2010. 